International Journal of Difference Equations (IJDE).

ISSN 0973-6069, Volume 16, Number 1, (2021). 47-57

CResearch India Publications

https://dx.doi.org/10.37622/IJDE/16.1.2021.47-57

\title{
Results on the Existence of Solutions of Sobolev-Type Volterra-Fredholm Integro-Differential Equations
}

\author{
Yogita M. Ahire ${ }^{1}$, Nedal M. Mohammed ${ }^{2}$ and Ahmed A. Hamoud*3 \\ ${ }^{1}$ Department of Applied Science, PVG'S College of Engineering, Nashik, \\ Maharashtra, India. \\ ${ }^{2}$ Department of Computer Science, Taiz University, Taiz, Yemen. \\ ${ }^{3}$ Department of Mathematics, Taiz University, Taiz, Yemen.
}

\begin{abstract}
In this study, the semigroup theory and the Schauder fixed point theorem are applied to prove the existence and uniqueness of mild and strong solutions of a nonlinear Volterra-Fredholm integro-differential equation of Sobolev type with nonlocal condition.
\end{abstract}

Keywords Volterra-Fredholm integro-differential equation; nonlocal condition; mild and strong solutions; fixed point technique.

Mathematics Subject Classification (2010): 45J05, 34A12, 47H10.

\section{Introduction}

Integro-differential equations arise in many areas of science and technology, specifically whenever a deterministic relation involving some continuously varying quantities and their rates of change in space and/or time are known or postulated [1-3,13,15-20]. The problem of existence of solutions of evolution equations with nonlocal conditions in Banach spaces has been studied first by Byszewski [10]. In that paper he has established the existence and uniqueness of mild, strong and classical solutions of the following nonlocal Cauchy problem:

$$
\left\{\begin{array}{l}
u^{\prime}(t)=-A u(t)+f(t, u(t)), \quad t \in\left(t_{0}, t_{0}+a\right] \\
u\left(t_{0}\right)+g\left(t_{1}, t_{2}, \ldots, t_{p}, u(.)\right)=u_{0}
\end{array}\right.
$$

*E-mail: ahmed.hamoud@taiz.edu.ye 
where $-A$ is the infinitesimal generator of a $C_{0}$ semigroup $T(t)$ on a Banach space $X, 0 \leq t_{0}<t_{1}<t_{2}<\ldots<t_{p} \leq t_{0}+a, a>0, u_{0} \in X$ and $f:\left[t_{0}, t_{0}+a\right] \times X \longrightarrow$ $X, g:\left[t_{0}, t_{0}+a\right]^{p} \times X \longrightarrow X$ are given functions. Subsequently several authors have investigated the same type of problem to different classes of abstract differential equations in Banach spaces [4-7, 11,14,21-31]. Brill [9] and Showalter [30] established the existence of solutions of semilinear evolution equations of Sobolev type in Banach spaces. This type of equations arise in various applications such as in the flow of fluid through fissured rocks [8], thermodynamics [12] and shear in second order fluids [22].

The purpose of this paper is to prove the existence of mild and strong solutions for Volterra-Fredholm integro-differential equation of Sobolev type with nonlocal condition of the form

$$
\begin{gathered}
(B u(t))^{\prime}+A u(t)=f(t, u(t))+\int_{0}^{t} g(t, s, u(s)) d s+\int_{0}^{a} h(t, s, u(s)) d s, \\
u(0)+\sum_{k=1}^{p} c_{k} u\left(t_{k}\right)=u_{0}
\end{gathered}
$$

where $0 \leq t_{1}<t_{2}<\ldots<t_{p} \leq a, B$ and $A$ are linear operators with domains contained in a Banach space $X$ and ranges contained in a Banach space $Y$ and the nonlinear operators $f: I \times X \longrightarrow Y$ and $g, h: \Delta \times X \longrightarrow Y$ are given. Here $I=[0, a]$ and $\Delta=\{(s, t): 0 \leq s \leq t \leq a\}, t \in J:=(0, a]$.

\section{Auxiliary results}

In order to prove our main theorem we assume certain conditions on the operators $A$ and $B$. Let $X$ and $Y$ be Banach spaces with norm $|$.$| and \|$.$\| respectively. The operators$ $A: D(A) \subset X \longrightarrow Y$ and $B: D(B) \subset X \longrightarrow Y$ satisfy the following hypothesis:

$\left(H_{1}\right) \quad A$ and $B$ are closed linear operators,

$\left(H_{2}\right) D(B) \subset D(A)$ and $B$ is bijective,

$\left(H_{3}\right) B^{-1}: Y \longrightarrow D(B)$ is bijective.

From the above fact and the closed graph theorem imply the boundedness of the linear operator $A B^{-1}: Y \longrightarrow Y$. Further $-A B^{-1}$ generates a uniformly continuous semigroup $T(t), t \geq 0$ and so $\max _{t \in I}\|T(t)\|$ is finite. We denote $M=$ $\max _{t \in I}\|T(t)\|, R=\left\|B^{-1}\right\|$. Let $B_{r}=\{x \in X:|x| \leq r\}$ and $c=\sum_{k=1}^{p}\left|c_{k}\right|$. 
Results on the Existence of Solutions of Sobolev-Type Volterra-Fredholm...

In this paper, we assume that there exists an operator $E$ on $D(E)=X$ given by the formula

$$
E=\left[I+\sum_{k=1}^{p} c_{k} B^{-1} T\left(t_{k}\right) B\right]^{-1}
$$

and $E u_{0} \in D(B)$

$E \int_{0}^{t_{k}} B^{-1} T\left(t_{k}-s\right)\left[f(s, u(s))+\int_{0}^{s} g(s, \tau, u(\tau)) d \tau+\int_{0}^{a} h(s, \tau, u(\tau)) d \tau\right] d s \in D(B)$.

The existence of $E$ can be observed from the following fact [11]. Suppose that $\{T(t)\}$ is a $C_{0}$ semigroup of operators on $X$ such that $\left\|B^{-1} T\left(t_{k}\right) B\right\| \leq C e^{-\delta t_{k}}(k=$ $1,2, \ldots, p)$ where $\delta$ is a positive constant and $C \geq 1$. If $\sum_{k=1}^{p}\left|c_{k}\right| e^{-\delta t_{k}}<1 / C$ then $\left\|\sum_{k=1}^{p} c_{k} B^{-1} T\left(t_{k}\right) B\right\|<1$. So such an operator $E$ exists on $X$.

Definition 2.1 [28] A continuous solution u of the integral equation

$$
\begin{aligned}
u(t) & =B^{-1} T(t) B E u_{0}-\sum_{k=1}^{p} c_{k} B^{-1} T(t) B E \int_{0}^{t_{k}} B^{-1} T\left(t_{k}-s\right) \\
& \times\left[f(s, u(s))+\int_{0}^{s} g(s, \tau, u(\tau)) d \tau+\int_{0}^{a} h(s, \tau, u(\tau)) d \tau\right] d s+\int_{0}^{t} B^{-1} T(t-s) \\
& \times\left[f(s, u(s))+\int_{0}^{s} g(s, \tau, u(\tau)) d \tau+\int_{0}^{a} h(s, \tau, u(\tau)) d \tau\right] d s
\end{aligned}
$$

is said to be a mild solution of the problem (2)-(3) on I

Definition 2.2 [28] A function $u$ is said to be a strong solution of the problem (2)-(3) on $I$ if $u$ is differentiable almost everywhere on $I, u^{\prime} \in L^{1}(I, X), u(0)+\sum_{k=1}^{p} c_{k} u\left(t_{k}\right)=$ $u_{0}$ and

$$
(B u(t))^{\prime}+A u(t)=f(t, u(t))+\int_{0}^{t} g(t, s, u(s)) d s+\int_{0}^{a} h(t, s, u(s)) d s, \text { a.e on } I \text {. }
$$

Remark. A mild solution of the nonlocal Cauchy problem (2)-(3) satisfies the condition (3). From (4)

$$
\begin{aligned}
u(0) & =E u_{0}-\sum_{k=1}^{p} c_{k} E \int_{0}^{t_{k}} B^{-1} T\left(t_{k}-s\right)[f(s, u(s)) \\
& \left.+\int_{0}^{s} g(s, \tau, u(\tau)) d \tau+\int_{0}^{a} h(s, \tau, u(\tau)) d \tau\right]
\end{aligned}
$$


and

$$
\begin{aligned}
u\left(t_{i}\right) & =B^{-1} T\left(t_{i}\right) B E u_{0}-\sum_{k=1}^{p} c_{k} B^{-1} T\left(t_{i}\right) B E \int_{0}^{t_{k}} B^{-1} T\left(t_{k}-s\right) \\
& \times\left[f(s, u(s))+\int_{0}^{s} g(s, \tau, u(\tau)) d \tau+\int_{0}^{a} h(s, \tau, u(\tau)) d \tau\right] d s+\int_{0}^{t_{i}} B^{-1} T\left(t_{i}-s\right) \\
& \times\left[f(s, u(s))+\int_{0}^{s} g(s, \tau, u(\tau)) d \tau+\int_{0}^{a} h(s, \tau, u(\tau)) d \tau\right] d s .
\end{aligned}
$$

Therefore

$$
\begin{aligned}
& u(0)+\sum_{i=1}^{p} c_{i} u\left(t_{i}\right) \\
= & {\left[I+\sum_{i=1}^{p} c_{i} B^{-1} T\left(t_{i}\right) B\right] E u_{0} } \\
- & {\left[I+\sum_{i=1}^{p} c_{k} B^{-1} T\left(t_{i}\right) B\right] \sum_{k=1}^{p} c_{k} E \int_{0}^{t_{k}} B^{-1} T\left(t_{k}-s\right) } \\
\times & {\left[f(s, u(s))+\int_{0}^{s} g(s, \tau, u(\tau)) d \tau+\int_{0}^{a} h(s, \tau, u(\tau)) d \tau\right] d s+\sum_{i=1}^{p} c_{i} } \\
\times & \int_{0}^{t_{i}} B^{-1} T\left(t_{i}-s\right)\left[f(s, u(s))+\int_{0}^{s} g(s, \tau, u(\tau)) d \tau+\int_{0}^{a} h(s, \tau, u(\tau)) d \tau\right] d s \\
= & u_{0}-\sum_{k=1}^{p} c_{k} \int_{0}^{t_{k}} B^{-1} T\left(t_{k}-s\right) \\
\times & {\left[f(s, u(s))+\int_{0}^{s} g(s, \tau, u(\tau)) d \tau+\int_{0}^{a} h(s, \tau, u(\tau)) d \tau\right] d s+\sum_{i=1}^{p} c_{i} } \\
\times & \int_{0}^{t_{i}} B^{-1} T\left(t_{i}-s\right)\left[f(s, u(s))+\int_{0}^{s} g(s, \tau, u(\tau)) d \tau+\int_{0}^{a} h(s, \tau, u(\tau)) d \tau\right] d s \\
= & u_{0} .
\end{aligned}
$$

Further assume that,

$\left(H_{4}\right) g, h: \Delta \times B_{r} \longrightarrow Y$ is continuous in t and there exist two constants $K_{1}, K_{2}>0$ such that $\|g(t, s, u)\| \leq K_{1},\|h(t, s, u)\| \leq K_{2}$ for $(s, t) \in \Delta$ and $u \in B_{r}$

$\left(H_{5}\right) f: I \times B_{r} \longrightarrow Y$ is continuous in $t$ on $I$ and there exists a constant $L>0$ such that $\|f(t, u)\| \leq L$ for $t \in I$, and $u \in B_{r}$

$\left(H_{6}\right) R M\left\|B E u_{0}\right\|+\left(R^{2} M^{2} a\|B E\| c+R M a\right)\left(L+\left(K_{1}+K_{2}\right) a\right) \leq r$. 


\section{Main Results}

Theorem 3.1 If the assumptions $\left(H_{1}\right) \sim\left(H_{6}\right)$ hold, then the problem (2)-(3) has a mild solution on $I$.

Proof. Let $Z=C(I, X)$ and $Z_{0}=\left\{u \in Z: u(t) \in B_{r}, t \in I\right\}$. Clearly, $Z_{0}$ is a bounded closed convex subset of $Z$. We define a mapping $F: Z_{0} \longrightarrow Z_{0}$ by

$$
\begin{aligned}
(F u)(t) & =B^{-1} T(t) B E u_{0}-\sum_{k=1}^{p} c_{k} B^{-1} T(t) B E \int_{0}^{t_{k}} B^{-1} T\left(t_{k}-s\right) \\
& \times\left[f(s, u(s))+\int_{0}^{s} g(s, \tau, u(\tau)) d \tau+\int_{0}^{a} h(s, \tau, u(\tau)) d \tau\right] d s+\int_{0}^{t} B^{-1} T(t-s) \\
& \times\left[f(s, u(s))+\int_{0}^{s} g(s, \tau, u(\tau)) d \tau+\int_{0}^{a} h(s, \tau, u(\tau)) d \tau\right] d s, t \in I .
\end{aligned}
$$

Obviously $F$ is continuous and maps $Z_{0}$ into itself. Moreover, $F$ maps $Z_{0}$ into a precompact subset of $Z_{0}$. Note that the set $Z_{0}(t)=\left\{(F u)(t): u \in Z_{0}\right\}$ is precompact in $X$, for every fixed $t \in I$. We shall show that $F\left(Z_{0}\right)=S=\left\{F u: u \in Z_{0}\right\}$ is an equicontinuous family of functions. For $0<s<t$, we have

$$
\begin{aligned}
& \|(F u)(t)-(F u)(s)\| \\
\leq & \left\|B^{-1} T(t)-T(s) B E u_{0}\right\|+R^{2} M a\|B E\|\left(L+\left(K_{1}+K_{2}\right) a\right) \sum_{k=1}^{p} c_{k}\|T(t)-T(s)\| \\
+ & \int_{0}^{t}\left\|B^{-1}\right\|\|T(t-\theta)-T(s-\theta)\|\left[\|f(\theta, u(\theta))\|+\int_{0}^{\theta} g(\theta, \tau, u(\tau)) d \tau+\int_{0}^{a} h(\theta, \tau, u(\tau)) d \tau\right] d \theta \\
+ & \int_{0}^{t}\left\|B^{-1}\right\|\|T(s-\theta)\|\left[\|f(\theta, u(\theta))\|+\int_{0}^{\theta} g(\theta, \tau, u(\tau)) d \tau+\int_{0}^{a} h(\theta, \tau, u(\tau)) d \tau\right] d \theta \\
\leq & \left(R\left\|B E u_{0}\right\|+R^{2} M a\|B E\|\left(L+\left(K_{1}+K_{2}\right) a\right) c\right)\|T(t)-T(s)\| \\
+ & R\left(L+\left(K_{1}+K_{2}\right) a\right) \int_{0}^{t}\left\|B^{-1}\right\|\|T(t-\theta)-T(s-\theta)\| d \theta+R M\left(L+\left(K_{1}+K_{2}\right) a\right)|t-s| .
\end{aligned}
$$

The right hand side of the above inequality is independent of $u \in Z_{0}$ and tends to zero as $s \longrightarrow t$ as a consequence of the continuity of $T(t)$ in the uniform operator topology for $t>0$. It is also clear that $S$ is bounded in $Z$. Thus by Arzela $a^{\lrcorner}$Ascoli'stheorem, $S$ is precompact. Hence by the Schauder fixed point theorem, $F$ has a fixed point in $Z_{0}$ and any fixed point of $F$ is a mild solution of (2)-(3) on $I$ such that $u(t) \in X$ for $t \in I$. Next we prove that the problem (2)-(3) has a strong solution.

Theorem 3.2 . Assume that

(i) Conditions $\left(H_{1}\right) \sim\left(H_{6}\right)$ hold; 
(ii) $Y$ is a reflexive Banach space with norm $\|$.$\| ;$

(iii) $f: I \times B_{r} \longrightarrow Y$ is Lipschitz continuous in $t$ that is, there exists a constant $L_{1}>0$ such that $\|f(t, u)-f(s, v)\| \leq L_{1}[|t-s|+\|u-v\|]$ for $s, t \in I$ and $u, v \in B_{r}$;

(iv) $g, h: \Delta \times B_{r} \longrightarrow Y$ is Lipschitz continuous in t that is, there exists a constant $L_{2}>0$ such that $\|g(t, \tau, u)-g(s, \tau, u)\| \leq L_{g}|t-s|,\|h(t, \tau, u)-h(s, \tau, u)\| \leq L_{h}|t-s|$ for $(t, \tau),(s, t) \in \Delta$ and $u \in B_{r}$;

(v) $E u_{0} \in D\left(A B^{-1}\right)$ and $E \int_{0}^{t_{k}} B^{-1} T\left(t_{k}-s\right)\left[f(s, u(s))+\int_{0}^{s} g(s, \tau, u(\tau)) d \tau+\int_{0}^{a} h(s, \tau, u(\tau)) d \tau\right] d s \in D(B) ;$

(vi) $u$ is the unique mild solution of the problem (2)-(3). Then $u$ is a unique strong solution of the problem (2)-(3) on I.

Proof. Since all the assumptions of Theorem 3.1 are satisfied, then the problem (2)-(3) has a mild solution belonging to $C\left(I, B_{r}\right)$. By assumption $(v i), u$ is the unique mild solution of the problem (2)-(3). Now, we shall show that $u$ is a unique strong solution of the problem (2)-(3) on $I$.

For any $t \in I$, we have

$$
\begin{aligned}
& u(t+h)-u(t) \\
= & B^{-1}[T(t+h)-T(t)] B E u_{0}-\sum_{k=1}^{p} c_{k} B^{-1}[T(t+h)-T(t)] B E \\
\times & \int_{0}^{t_{k}} B^{-1} T\left(t_{k}-s\right)\left[f(s, u(s))+\int_{0}^{s} g(s, \tau, u(\tau)) d \tau+\int_{0}^{a} h(s, \tau, u(\tau)) d \tau\right] d s \\
+ & \int_{0}^{h} B^{-1} T(t+h-s)\left[f(s, u(s))+\int_{0}^{s} g(s, \tau, u(\tau)) d \tau+\int_{0}^{a} h(s, \tau, u(\tau)) d \tau\right] d s \\
+ & \int_{0}^{t+h} B^{-1} T(t+h-s)\left[f(s, u(s))+\int_{0}^{s} g(s, \tau, u(\tau)) d \tau+\int_{0}^{a} h(s, \tau, u(\tau)) d \tau\right] d s \\
- & \int_{0}^{t} B^{-1} T(t-s)\left[f(s, u(s))+\int_{0}^{s} g(s, \tau, u(\tau)) d \tau+\int_{0}^{a} h(s, \tau, u(\tau)) d \tau\right] d s
\end{aligned}
$$




$$
\begin{aligned}
& =B^{-1} T(t)[T(h)-I] B E u_{0}-\sum_{k=1}^{p} c_{k} B^{-1}[T(t+h)-T(t)] B E \\
& \times \int_{0}^{t_{k}} B^{-1} T\left(t_{k}-s\right)\left[f(s, u(s))+\int_{0}^{s} g(s, \tau, u(\tau)) d \tau \int_{0}^{a} h(s, \tau, u(\tau)) d \tau\right] d s \\
& +\int_{0}^{h} B^{-1} T(t+h-s)\left[f(s, u(s))+\int_{0}^{s} g(s, \tau, u(\tau)) d \tau+\int_{0}^{a} h(s, \tau, u(\tau)) d \tau\right] d s \\
& +\quad \int_{0}^{t} B^{-1} T(t-s)[f(s+h, u(s+h))-f(s, u(s))] d s \\
& +\int_{0}^{t} B^{-1} T(t-s)\left[\int_{0}^{s+h} g(s+h, \tau, u(\tau)) d \tau-\int_{0}^{s} g(s, \tau, u(\tau)) d \tau\right] d s \\
& +\quad \int_{0}^{t} B^{-1} T(t-s)\left[\int_{0}^{a} h(s+h, \tau, u(\tau)) d \tau-\int_{0}^{a} h(s, \tau, u(\tau)) d \tau\right] d s .
\end{aligned}
$$

Using our assumptions we observe that

$$
\begin{aligned}
& \|u(t+h)-u(t)\| \\
\leq & R\left\|B E u_{0}\right\| M h\left\|A B^{-1}\right\|+c M^{2} R^{2} a\|B E\|\left(L+\left(K_{1}+K_{2}\right) a\right) h\left\|A B^{-1}\right\| \\
+ & h R M\left(L+\left(K_{1}+K_{2}\right) a\right)+R M \int_{0}^{t} L_{1}[h+\|u(s+h)-u(s)\|] d s \\
+ & R M \int_{0}^{t}\left[\int_{0}^{s}\|g(s+h, \tau, u)-g(s, \tau, u)\| d \tau+\int_{0}^{s+h}\|g(s+h, \tau, u)\| d \tau\right] d s \\
+ & R M \int_{0}^{t}\left[\int_{0}^{a}\|h(s+h, \tau, u)-h(s, \tau, u)\| d \tau+\int_{0}^{a}\|h(s+h, \tau, u)\| d \tau\right] d s \\
\leq & R\left\|B E u_{0}\right\| M h\left\|A B^{-1}\right\|+\left[c M^{2} R^{2} a\|B E\| h\|\| A B^{-1} \|+h R M\right]\left(L+\left(K_{1}+K_{2}\right) a\right) \\
+ & R M L_{1} \int_{0}^{t}[h+\|u(s+h)-u(s)\|] d s+R M a h\left(\left(K_{1}+K_{2}\right)+\left(L_{g}+L_{h}\right) a\right) \\
\leq & P h+Q \int_{0}^{t} \mid u(s+h)-u(s) \| d s,
\end{aligned}
$$

where

$$
\begin{gathered}
P=R\left\|B E u_{0}\right\| M\left\|A B^{-1}\right\|+c M^{2} R^{2} a\|B E\|\left\|A B^{-1}\right\|+R M\left(L+\left(K_{1}+K_{2}\right) a\right) \\
+M R L_{1} a+R M\left(K_{1}+K_{2}\right) a+R M\left(L_{g}+L_{h}\right) a^{2}
\end{gathered}
$$

and $Q=R M L_{1}$. By Gronwallis inequality

$$
\|u(t+h)-u(t)\| \leq P h e^{Q}, \text { for } t \in J .
$$

Therefore, $u$ is Lipschitz continuous on $I$. The Lipschitz continuity of $u$ on $I$ combined with (iii) and (iv) imply that

$$
t \longrightarrow f(t, u(t)), t \longrightarrow \int_{0}^{t} g(t, s, u(s)) d s \text { and } t \longrightarrow \int_{0}^{a} h(t, s, u(s)) d s, t \in(0, a]
$$


are Lipschitz continuous on $I$. Using the Corollary 2.11 [28] and the definition of strong solution we observe that the linear Cauchy problem:

$$
\begin{gathered}
(B v(t))^{\prime}+A v(t)=f(t, u(t))+\int_{0}^{t} g(t, s, u(s)) d s+\int_{0}^{a} h(t, s, u(s)) d s, t \in(0, a], \\
v(0)=u_{0}-\sum_{k=1}^{p} c_{k} u\left(t_{k}\right),
\end{gathered}
$$

has a unique strong solution $v$ satisfying the equation

$$
\begin{aligned}
v(t) & =B^{-1} T(t) B E v(0)+\int_{0}^{t} B^{-1} T(t-s) \\
& \times\left[f(s, u(s))+\int_{0}^{s} g(s, \tau, u(\tau)) d \tau+\int_{0}^{a} h(s, \tau, u(\tau)) d \tau\right] d s, \quad t \in I
\end{aligned}
$$

Now, we will show that $v(t)=u(t)$ for $t \in I$. Observe that

$$
\begin{aligned}
v(0) & =u(0)=E u_{0}-\sum_{k=1}^{p} c_{k} E \int_{0}^{t_{k}} B^{-1} T\left(t_{k}-s\right) \\
& \times\left[f(s, u(s))+\int_{0}^{s} g(s, \tau, u(\tau)) d \tau+\int_{0}^{a} h(s, \tau, u(\tau)) d \tau\right] d s
\end{aligned}
$$

So

$$
\begin{aligned}
B^{-1} T(t) B E v(0) & =B^{-1} T(t) B E u_{0}-\sum_{k=1}^{p} c_{K} B^{-1} T(t) B E \int_{0}^{t_{k}} B^{-1} T\left(t_{k}-s\right) \\
& \times\left[f(s, u(s))+\int_{0}^{s} g(s, \tau, u(\tau)) d \tau+\int_{0}^{a} h(s, \tau, u(\tau)) d \tau\right] d s
\end{aligned}
$$

Substituting this in the equation (6) we see that $v(t)=u(t)$. Consequently, $u$ is a strong solution of the problem (2)-(3) on $I$.

\section{Example}

Consider the following differential equation

$$
\begin{gathered}
\frac{\partial}{\partial t}\left(z(t, x)-z_{x x}(t, x)\right)=\mu(t, z(t, x))+\int_{0}^{t} \eta_{1}(t, s, z(t, x)) d s+\int_{0}^{\pi} \eta_{2}(t, s, z(t, x)) d s, \\
z(t, 0)=z(t, \pi)=0, t \in J,
\end{gathered}
$$




$$
z(0, x)+\sum_{k=1}^{p} z\left(t_{k}, x\right)=z_{0}(x), x \in J:=[0, \pi], 0<t_{1}<t_{2}<\ldots<t_{p} \leq a .
$$

Let us take $X=Y=L^{2}[0, \pi]$. Define the operators $A: D(A) \longrightarrow X \longrightarrow Y$, $B: D(B) \longrightarrow X \longrightarrow Y$ by

$$
\begin{gathered}
A z=-z_{x x}, \\
B z=z-z_{x x},
\end{gathered}
$$

respectively, where each domain $D(A), D(B)$ is given by

$$
\left\{z \in X: z, z_{x} \text { are absolutely continuous, } z_{x x} \in X, z(0)=z(\pi)=0\right\} .
$$

Define the operators $f: J \times X \longrightarrow Y, g, h: \Delta \times X \longrightarrow Y$ by $f(t, z)(x)=\mu(t, z(t, x)), g(t, s, z)(x)=\eta_{1}(t, s, z(t, x)), h(t, s, z)(x)=\eta_{2}(t, s, z(t, x))$, and satisfy the conditions $\left(H_{4}\right)$ and $\left(H_{5}\right)$ on a bounded closed set $B_{r} \subset X$. Here $r$ satisfies the condition $\left(H_{6}\right)$ Then the above problem (7) can be formulated abstractly as

$$
(B z(t))^{\prime}+A z(t)=f(t, z)+\int_{0}^{t} g(t, s, z(s)) d s+\int_{0}^{\pi} h(t, s, z(s)) d s, \text { a.e on } J .
$$

Also, $A$ and $B$ can be written as

$$
\begin{gathered}
A z=\sum_{n=1}^{\infty} n^{2}\left\langle z, z_{n}\right\rangle z_{n}, \quad z \in D(A) \\
B z=\sum_{n=1}^{\infty}\left(1+n^{2}\right)\left\langle z, z_{n}\right\rangle z_{n} \quad z \in D(B)
\end{gathered}
$$

where $z_{n}(x)=\sqrt{2 / \pi} \sin n x, n=1,2, \ldots$ is the orthogonal set of eigenfunctions of $A$. Furthermore, for $z \in X$ we have

$$
\begin{gathered}
B^{-1} z=\sum_{n=1}^{\infty} \frac{1}{\left(1+n^{2}\right)}\left\langle z, z_{n}\right\rangle z_{n}, \\
-A B^{-1} z=\sum_{n=1}^{\infty} \frac{-n^{2}}{\left(1+n^{2}\right)}\left\langle z, z_{n}\right\rangle z_{n} \\
T(t) z=\sum_{n=1}^{\infty} e^{-n^{2} t /\left(1+n^{2}\right)}\left\langle z, z_{n}\right\rangle z_{n} .
\end{gathered}
$$

It is easy to see that $-A B^{-1}$ generates a strongly continuous semigroup $T(t)$ on $Y$ and $T(t)$ is compact such that $\|T(t)\| \leq e^{-t}$ for each $t>0$. For this $T(t), B, B^{-1}$ we assume that the operator $E$ exists. So all the conditions of the above theorem are satisfied. Hence the equation (7) with nonlocal condition (8) has a mild solution. 


\section{References}

[1] Alasadi, I. and Hamoud, A. Existence and stability results for fractional Volterra-Fredholm integro-dierential equation with mixed conditions, Advances in Dynamical Systems and Applications, 16(1) (2021), 217-236.

[2] Bhadane, P., Hamoud, A. and Ghadle, K. Approximate solution of fractional BlackSchole's European option pricing equation by using ETHPM, Nonlinear Functional Analysis and Applications, 25(2) (2020), 331-344.

[3] Bani Issa, M., Hamoud, A. and Ghadle, K. Numerical solutions of fuzzy integrodifferential equations of the second kind, Journal of Mathematics and Computer Science, 23 (2021), 67-74.

[4] Balachandran, K. and Chandrasekaran, M. Existence of solutions of nonlinear integrodifferential equations with nonlocal condition, J. Appl. Math. Stoch. Anal. 10(1997) 279288.

[5] Balachandran, K. and Chandrasekaran, M. Existence of solutions of a delay differential equation with nonlocal condition, Indian J. Pure. Appl. Math. 27(1996) 443-449.

[6] Balachandran, K., Park, D.G. and Kwun, Y.C. Nonlinear integro-differential equations of Sobolev type with nonlocal conditions in Banach spaces, Comm. Korean Math. Soc. 14(1999) 223-231.

[7] Balachandran, K. and Ilamaran, S. Existence and uniqueness of mild and strong solutions of a Volterra integro-differential equation with nonlocal conditions, Tamkang J. Math. 28(1997) 93-100.

[8] Barenblatt, G., Zheltov, I. and Kochina, I. Basic concepts in the theory of seepage of homogeneous liquids in fissured rocks, J. Appl. Math. Mech. 24(1960) 1286-1303.

[9] Brill, H. A semilinear Sobolev evolution equation in Banach space, J. Diff. Eq. 24(1977) 412-425.

[10] Byszewski, L. Theorems about the existence and uniqueness of solutions of a semilinear evolution nonlocal Cauchy problem, J. Math. Anal. Appl. 162(1991) 494-505.

[11] Byszewski, L. Applications of properties of the right hand sides of evolution equations to an investigation of nonlocal evolution problems, Nonlinear Anal. 33(1998) 413-426.

[12] Chen, P.J. and Curtin, M. E. On a theory of heat conduction in volving two temperatures, Z. Angew. Math. Phys. 19(1968) 614-627.

[13] Hamoud, A. and Ghadle, K. Modified Adomian decomposition method for solving fuzzy Volterra-Fredholm integral equations, J. Indian Math. Soc., 85(1-2) (2018), 52-69.

[14] Hamoud, A. Uniqueness and stability results for Caputo fractional Volterra-Fredholm integro-differential equations, J. Sib. Fed. Univ. Math. Phys., 14(3)(2021) 313-325.

[15] Hadeed, H.S., Hamad, A.S. and Hamoud, A.A. Numerical Iterative Methods for Solving Nonlinear Volterra-Fredholm Integral Equations, Advances in Dynamical Systems and Applications, 16(2)(2021) 535-545.

[16] Hamoud, A. and Ghadle, K. Some new existence, uniqueness and convergence results for fractional Volterra-Fredholm integro-differential equations, J. Appl. Comput. Mech., 5(1) (2019) 58-69.

[17] Hamoud, A. and Ghadle, K. Existence and uniqueness of solutions for fractional mixed Volterra-Fredholm integro-differential equations, Indian J. Math., 60(3) (2018) 375-395. 
[18] Hamoud, A. and Ghadle, K. Existence and uniqueness of the solution for VolterraFredholm integro-differential equations, J. Siberian Federal University. Math. Phys., 11(6) (2018) 692-701.

[19] Hamoud, A. and Ghadle, K. Usage of the homotopy analysis method for solving fractional Volterra-Fredholm integro-differential equation of the second kind, Tamkang J. Math., 49 (4) (2018) 301-315.

[20] Hamoud, A. and Ghadle, K. Modified Laplace decomposition method for fractional Volterra-Fredholm integro-differential equations, Journal of Mathematical Modeling, 6(1) (2018) 91-104.

[21] Hamoud, A. Existence and uniqueness of solutions for fractional neutral VolterraFredholm integro-differential equations, Advances in the Theory of Nonlinear Analysis and its Application, 4(4)(2020) 321-331.

[22] Huilgol, R. A second order fluid of the differential type, Internat. J. Nonlinear Mech. 3(1968) 471-482.

[23] Hussain, K., Hamoud, A. and Mohammed, N. Some new uniqueness results for fractional integro-differential equations, Nonlinear Funct. Anal. Appl., 24(4) (2019) 827-836.

[24] Jackson,D. Existence and uniqueness of solutions to semilinear nonlocal parabolic equations, J. Math.Anal. Appl. 172(1993) 256-265.

[25] Lightbourne, J.H. and Rankin, S.M. A partial functional differential equation of Sobolev type, J. Math. Anal. Appl. 93(1983) 328-337.

[26] Lin, Y. and LiuJ, U. Semilinear integro-differential equations with nonlocal Cauchy problem, Nonlinear Anal. TMA. 26(1996) 1023-1033.

[27] Ntouyas, S.K. and Tsamatos, P.Ch. Global existence of semilinear evolution equation with nonlocal conditions, J. Math. Anal. Appl. 210(1997) 679-687.

[28] Pazy, A. Semigroups of linear operators anda pplications to partial differential equations, (New York:Springer-Verlag)(1983).

[29] Sharif, A. and Hamoud, A. On $\psi$-Caputo fractional nonlinear Volterra-Fredholm integrodifferential equations, Discontinuity, Nonlinearity, and Complexity, 11(1) (2022), 97-106.

[30] Showalter, R.E. Existence and representation theorem for a semilinear Soboleve quationin Banach space, SIAMJ. Math. Anal. 3(1972) 527-543.

[31] Younis, M.F., Abed, A.M. and Hamoud, A.A. Existence and uniqueness results for bvp of nonlinear fractional Volterra-Fredholm integro-differential equation, Advances in Dynamical Systems and Applications, 16(2)(2021) 457-467. 
